Methods 14 patients with active rheumatoid arthritis and unresponsive to at least 3 DMARDs were treated with methotrexate and infliximab. Before infliximab infusion, weeks 0, 2,6,14 and 22, a physical examination, DAS 28 and blood test, which included RF, ANA, immunoglobulin quantification C3 and C4 were done.

Results At the baseline visit 5/14 (35,71\%) patients had positive ANA test,2 (1/40), 1 (1/80), 1 (1/160), 1 (1/640). After the fifth infusion $8 / 14(57,14 \%)$ had a positive ANA test. Three patients who initially were ANA negative showed a titre of $1 / 160,1 / 320$ and $1 / 640$. Three out of 5 patients which previously had a positive test, showed an increase in the ANA titre, $1 / 40$ to $1 / 80,1 / 40$ to $1 / 320$ and $1 / 640$ to $1 / 1280$. After the fourth infusion of infliximab, all patients with ANA positive had a Crithidia test performed. In all cases anti DNA were negative.

Conclusion In our experience, treatment with infliximab has shown an increase of the ANA titre in $21,42 \%$ of the patients. On the other hand, $21,4 \%$ of the patients with a previous negative test for ANA had a positive test after the fourth infusion. All cases were negative for anti-DNA antibodies.

\section{FRI0053 STEROIDS RESTORE THE IMPAIRED CERULOPLAMIN FERROXIDASE ACTIVITY IN SYNOVIAL FLUID OF RHEUMATOID ARTHRITIS}

V Livshitz, AM Nahir. Rheumatology, Faculty of Medicine - Technion, Haifa, Israel

\subsection{6/annrheumdis-2001.1182}

Background Ceruloplasmin (CP) is an acute phase reactant with significant antioxidative activity. In rheumatoid arthritis (RA), joint destruction continues in spite of high levels of CP in the synovial fluid (SF). The antioxidative activity of CP correlates with its ferroxidase I activity (FerrI). The biology role of the recently discovered ferroxidase II (FerrII) is unknown.

Objectives The present study was aimed to find the effect of drug therapy on CP levels and CP ferroxidase activity in RA SF and to compare it to SF nitrite levels, which indicate the degree of the inflammatory process.

Methods SF was collected from 75 RA patients who were:

- w/o drug therapy (UT).

- with NSAIDs and disease modifying drugs (DMD), mainly MTX who had no steroid in the last 3 months (NST).

- with low dose steroids $(=/<10 \mathrm{mg}$ Prednisone $)+$ DMD ST).

- SF of osteoarthritic (OA) patients served as control. For CP levels Ravin's method was used. ${ }^{1}$ For CP ferroxidase activity Johnson's method was used. ${ }^{2}$ For FerrII activity Johnson's method in the presence of sodium azide. FerrI activity was calculated: Total-FerrII. For nitrite levels Ding's method was used. ${ }^{3}$

Results Inspite of drug therapy and nitrite level similar to OA, $\mathrm{CP}$ and total ferroxidase activity remained high in all RA groups. FerrI/FerrII ratio was significantly abnrmal in RA without steroids. Low dose steroids restored FerrI/FerrII ratio to levels seen in OA.

Conclusion Low dose steroid therapy in RA did not reduce SF ceruloplasmin concentration, but restored FerrI/FerrII ratio to normal levels.

\section{REFERENCES}

1 Ravin HA. J Lab Clin Med. 1961;58:161-8

2 Johnson DA, et al. Clin Chem. 1967;13:142-9

3 Ding $\mathrm{AH}$, et al. J Immunol. 1998;141:2407-13
FRI0054 A LONG-TERM, OPEN-LABEL TRIAL OF THE SAFETY AND EFFICACY OF ETANERCEPT (25 MG TWICE WEEKLY) IN PATIENTS WITH RHEUMATOID ARTHRITIS (INTERIM ANALYSIS)

RF Willis, R Pedersen. The Etanercept European Investigators Network, Wyeth-Ayerst Research, Radnor, USA

10.1136/annrheumdis-2001.1183

Background Several double-blind, placebo-controlled studies previously demonstrated that etanercept was safe and efficacious in treating rheumatoid arthritis (RA) patients who had an inadequate response to disease-modifying antirheumatic drugs (DMARDs).

Objectives To evaluate the long-term safety and efficacy of etanercept in patients who completed prior double-blind clinical studies comparing etanercept to placebo.

Methods As reported in previous updates, 549 patients entered this 4-year, open-label study and began treatment with etanercept $25 \mathrm{mg}$ twice weekly at 58 centres in Europe following completion of double-blind clinical studies. All patients previously had inadequate responses to DMARDs. Safety assessments were performed at regular intervals to determine the incidence of treatment emergent adverse events including malignancies and serious infections (those associated with hospitalisation and/or the administration of intravenous antibiotics). The numbers of painful and swollen joints were predefined as primary efficacy endpoints; other efficacy measures included ACR response rates and acute phase reactants. Efficacy was analysed with the last observation carried forward (LOCF).

Results Of the 549 patients initially enrolled, 437 (80\%) are currently active, 479 (87\%) completed 1 year, and 94 (17\%) completed 2 years. A total exposure of 927 patient-years has been accrued. The rate of withdrawal from the study was similar for efficacy- and tolerance-related reasons $(7 \%$ and $8 \%$, respectively). Adjusted for patient exposure, the most frequent adverse events were injection site reactions and upper respiratory infections. Rates of serious infections and malignancies have remained unchanged over the course of the study. Maintained efficacy was observed as demonstrated by a mean $71 \%$ and $72 \%$ reduction in painful and swollen joint counts, respectively. Similar to results measured at the early time points of this open-label trial, ACR 20,50 , and 70 response rates were determined to be $79 \%, 47 \%$, and $25 \%$, respectively, as patients approached two years of treatment.

Conclusion Following the accumulation of substantial exposure in patients with RA, etanercept demonstrates an acceptable safety profile and continues to provide significant and maintained clinical benefit.

\section{FRI0055 EXPERIENCE WITH COMMERCIAL REMICADE (INFLIXIMAB) AT A LARGE COMMUNITY-BASED RHEUMATOLOGY PRACTICE}

W Shergy, RM Phillips, RE Hunt, J Hernandez. Associate Clinical Professors of Medicine, University of Alabama/Huntsville and Rheumatology Associates of N. Alabama, Huntsville, USA

\subsection{6/annrheumdis-2001.1184}

Background Infliximab, a monoclonal antibody that binds with high affinity and specificity to TNF-alpha and neutralises its biologic activity, is approved for the reduction of the signs and symptoms of rheumatoid arthritis (RA). The Rheumatology 